\title{
Retinopatia solar após ritual religioso na cidade de Londrina
}

\author{
Occurrence of solar retinopathy after religious ritual in Londrina, Paraná, Brazil
}

\author{
Elaine Regina Ferraresi Sampaio ${ }^{1}$ \\ Antonio Marcelo Barbante Casella² \\ Michel Eid Farah ${ }^{3}$
}

\begin{tabular}{|l|}
\hline RESUMO \\
\hline Objetivo: Relato de uma série de casos de retinopatia solar após a observação \\
direta do sol durante ritual religioso na cidade de Londrina. Métodos: 24 \\
pacientes com alteração da visão após participarem de ritual religioso, foram \\
avaliados quanto à acuidade visual inicial e final, após 6 meses, refração, \\
biomicroscopia de mácula, retinografia, tela de Amsler e sensibilidade ao \\
contraste. Resultados: 24 casos foram relatados. Destes, 21 (87,5\%) eram \\
mulheres e 3(12,5\%) eram homens. A idade variou de 18 a 46 anos. Houve \\
acometimento de 43 olhos. A lesão foi bilateral em 19 (79,2\%) pacientes e \\
unilateral em 5(20,8\%). A acuidade visual inicial variou de 20/20 a 20/70 ea \\
final foi de 20/25, ou melhor, em 40 (93\%) olhos. Destes, 33 (76,7\%) olhos \\
alcançaram 20/20. Apenas 2(4,6\%) olhos ficaram com alteração da sensibi- \\
lidadeao contrastee3(6,9\%) ficaram com escotoma persistente. Conclusão: \\
Este é o maior relato de casos de retinopatia solar após ritual religioso. A \\
retinopatia solar apresenta evolução bastante favorável. No entanto, a \\
acuidade visual não apresenta recuperação completa da visão. Os pacientes \\
podem ficar com escotoma e metamorfopsia residual.
\end{tabular}

Descritores: Olho/patologia; Retina/patologia; Luz solar/efeitos adversos; Acuidade visual/efeitos de radiação; Comportamento ritualístico

\section{INTRODUÇÃO}

A retinopatia solar é uma afecção traumática da mácula causada pela exposição à energia radiante do sol por curto período de tempo ${ }^{(1-2)}$.

O perigo de se olhar para o sol é conhecido há séculos. Sócrates já advertia as pessoas a observarem o eclipse solar apenas pelo seu reflexo na água. Galileu, o pai da astronomia, relatava ter lesado seus olhos observando o sol através do telescópio ${ }^{(3)}$.

A observação do eclipse solar é a causa mais comum de retinopatia solar. Porém, esta entidade foi encontrada também em pilotos de avião, recrutas, astrônomos, banhistas, em adolescentes após ingestão de LSD, em esquizofrênicos, em peregrinos durante rituais religiosos onde os indivíduos são instados a olhar fixamente o sol para "enxergarem imagens celestiais" ou mesmo para "tornarem seus olhos mais fortes" ou por simples curiosidade ${ }^{(4-11)}$.

Durante os rituais religiosos, apesar de apresentarem um certo desconforto ocular, podem permanecer por períodos prolongados olhando para o sol e terem lesões significantes da visão.

Na cidade de Londrina, após uma jovem revelar ter conseguido contatos com Nossa Senhora, levou fervorosos fiéis, em busca de curas milagrosas, a participarem de um ritual religioso, na esperança de ver a Santa e tiveram a visão comprometida. Assim, fizemos o relato de casos de retinopatia solar após a observação direta do sol durante ritual religioso. 


\section{MÉTODOS}

No estudo foram relatados 24 pacientes com história de alteração da visão, após exposição solar durante ritual religioso na cidade de Londrina, no período de maio a setembro de 1999.

Os dados clínicos (sexo e idade), doenças oculares prévias, uso de medicações foram obtidos pela anamnese.

Os pacientes foram submetidos a exame oftalmológico completo e foram avaliados quanto a: acuidade visual inicial e final, refração, biomicroscopia de fundo, retinografia, tela de Amsler e teste de sensibilidade ao contraste.

A acuidade visual inicial foi obtida na primeira consulta com a melhor correção, o que ocorreu 3 a 7 dias após a exposição ao sol e a acuidade visual final considerada foi a encontrada 6 meses após a lesão.

O exame de fundo de olho foi realizado pela biomicroscopia de fundo com a lente de Volk de 78 dioptrias.

A retinografia foi realizada para o registro e medidas das lesões retinianas, pelo aparelho OIS (Ophthalmic Imaging System, 1024), na fase inicial e após 6 meses. Baseado na análise da retinografia, as alterações encontradas na fase inicial foram classificadas, de acordo com a intensidade da lesão em leve, moderada e grave. As ditas leves apresentavam um ponto amarelado foveal de coloração mais tênue e diâmetro menor que $180 \mu \mathrm{m}$. As moderadas eram mais amareladas e tinham um diâmetro maior que $180 \mu \mathrm{m}$. As lesões que apresentavam buraco lamelar foram consideradas graves.

Na fase tardia, os casos também foram classificados em leves, moderados e graves. Os leves apresentavam uma alteração do epitélio pigmentar menor que $180 \mu \mathrm{m}$ e os moderados, tinham diâmetro maior que $180 \mu \mathrm{m}$. Os graves apresentavam buraco macular verdadeiro.

A tela de Amsler e o teste de sensibilidade ao contraste foram utilizados para detecção de alterações maculares tardias.

Este estudo foi aprovado pelo Departamento de Ética da Universidade Estadual de Londrina os pacientes assinaram um termo de consentimento.

\section{RESULTADOS}

Foram examinados 24 pacientes com retinopatia solar aguda, após ritual religioso. Houve acometimento de 43 olhos e os dados referentes à idade, acuidade visual inicial e final, olho acometido, refração, tela de Amsler e teste de sensibilidade ao contraste estão apresentados na tabela 1 .

As queixas mais comuns referidas foram cefaléia, ofuscamento e diminuição da visão. Os pacientes quando interrogados quanto ao tempo de exposição ao sol, não sabiam precisar entre alguns segundos ou minutos. Nenhum usou óculos de sol ou estava fazendo uso de alguma medicação.

Dos 24 pacientes examinados, 21 (87,5\%) eram do sexo

\begin{tabular}{|c|c|c|c|c|c|c|c|c|c|}
\hline $\begin{array}{c}\text { Caso } \\
\mathbf{N}^{\circ}\end{array}$ & $\begin{array}{l}\text { Idade } \\
\text { (anos) }\end{array}$ & \multicolumn{2}{|c|}{ Inicial } & \multicolumn{2}{|c|}{ Final } & $\begin{array}{c}\text { Olho } \\
\text { acometido }\end{array}$ & & & Retinografia \\
\hline 1 & 31 & $20 / 40$ & $20 / 40$ & $20 / 20$ & $20 / 20$ & $\mathrm{AO}$ & $+0,75$ & $+0,75$ & $S$ \\
\hline 2 & 19 & $20 / 70$ & $20 / 20$ & $20 / 20$ & $20 / 20$ & OD & plano & plano & $S$ \\
\hline 3 & 38 & $20 / 25$ & $20 / 30$ & $20 / 25$ & $20 / 30$ & $\mathrm{AO}$ & $+0,25-0,25 \times 80$ & $-0,25 \times 90$ & $S$ \\
\hline 6 & 22 & $20 / 25$ & $20 / 25$ & $20 / 20$ & $20 / 20$ & $\mathrm{AO}$ & plano & plano & $S$ \\
\hline 7 & 36 & $20 / 20$ & $20 / 20$ & $20 / 20$ & $20 / 20$ & $\mathrm{AO}$ & $-0,25$ & $-0,25$ & $S$ \\
\hline 8 & 42 & $20 / 20$ & $20 / 20$ & $20 / 20$ & $20 / 20$ & $\mathrm{AO}$ & $+0,50$ & $+0,75$ & $S$ \\
\hline 9 & 27 & $20 / 400$ & $20 / 25$ & $20 / 400$ & $20 / 20$ & OE & $-0,50-0,25 \times 90$ & plano & $S$ \\
\hline 10 & 18 & $20 / 20$ & $20 / 20$ & $20 / 20$ & $20 / 20$ & $\mathrm{AO}$ & plano & plano & $S$ \\
\hline 15 & 36 & $20 / 40$ & $20 / 40$ & $20 / 20$ & $20 / 20$ & $\mathrm{AO}$ & $+0,25-0,25 \times 100$ & $+0,25$ & $S$ \\
\hline 16 & 37 & $20 / 25$ & $20 / 30$ & $20 / 25$ & $20 / 25$ & $\mathrm{AO}$ & $+0,25$ & $+0,50$ & $S$ \\
\hline 17 & 29 & $20 / 25$ & $20 / 25$ & $20 / 20$ & $20 / 20$ & $\mathrm{AO}$ & $-0,25 \times 75$ & $-0,25 \times 105$ & $S$ \\
\hline 18 & 43 & $20 / 40$ & $20 / 40$ & $20 / 30$ & $20 / 25$ & $\mathrm{AO}$ & $+0,25$ & $+0,25$ & $S$ \\
\hline 29 & 32 & $20 / 30$ & $20 / 30$ & $20 / 20$ & $20 / 20$ & $\mathrm{AO}$ & $+0,25-0,25 \times 180$ & $+0,25$ & $\mathrm{~S}$ \\
\hline 20 & 38 & $20 / 20$ & $20 / 25$ & $20 / 20$ & $20 / 20$ & $\mathrm{AO}$ & $+0,50$ & $+0,50$ & $S$ \\
\hline 21 & 41 & $20 / 40$ & $20 / 40$ & $20 / 20$ & $20 / 20$ & $\mathrm{AO}$ & $+0,25$ & $+0,25$ & $S$ \\
\hline 22 & 29 & $20 / 25$ & $20 / 20$ & $20 / 25$ & $20 / 20$ & $\mathrm{AO}$ & $-0,25 \times 180$ & $-0,25 \times 180$ & $S$ \\
\hline 23 & 25 & $20 / 20$ & $20 / 30$ & $20 / 20$ & $20 / 20$ & OE & $-0,25 \times 45$ & $+0,25$ & - \\
\hline 24 & 29 & $20 / 30$ & $20 / 30$ & $20 / 20$ & $20 / 20$ & $\mathrm{AO}$ & plano & plano & - \\
\hline
\end{tabular}


feminino e 3 (12,5\%) eram do sexo masculino. A idade variou entre 18 e 46 anos, com média de 31,6, desvio padrão de 8,1 e mediana de 32 anos.

O acometimento foi bilateral em 19 (79,2\%) pacientes e em 5 (20,8\%) foi unilateral. Destes, o olho direito foi acometido em 2 pacientes e olho esquerdo em 3 . O paciente $n^{\circ} 9$ por apresentar coriorretinite macular cicatrizada em um dos olhos, teve apenas o olho contralateral acometido. O paciente $\mathrm{n}^{\circ} 11$ apresentava ambliopia anisometrópica e teve o olho dominante acometido. $\mathrm{O}$ paciente $\mathrm{n}^{\circ} 12$ apresentava estrabismo convergente e ambliopia estrábica e teve também apenas o olho dominante acometido. Os pacientes $\mathrm{n}^{\circ} 2$ e $\mathrm{n}^{\circ} 23$ tiveram acometimento unilateral porque fecharam um dos olhos enquanto fitavam o sol (Tabela 1).

A acuidade visual inicial variou de 20/20 a 20/70 e a acuidade visual final obtida, após 6 meses da lesão, foi de 20/25, ou melhor, em 40 (93\%) olhos. Destes, 33 (76,7\%) olhos chegaram a 20/20 (Tabela 2).

O exame de refração foi realizado e o equivalente esférico dos 43 olhos variou de $-0,25$ a $+0,75$. A hipermetropia foi encontrada em 20 (46,6\%) olhos, astigmatismo em 11 (25,5\%), miopia em 3 (7\%) e emetropia em 9 (20,9\%).

A alteração encontrada à fundoscopia na fase inicial foi a presença de um ponto amarelado foveal e parafoveal que estava, em alguns casos, circundado por um halo eritematoso.

Os $40(100 \%)$ olhos que realizaram a retinografia foram analisados e classificados. Na análise das fotos, na fase inicial, foi observado que 27 (67,5\%) olhos apresentavam lesão leve (Figura 1) e 13 (32,5\%) olhos lesão moderada (Figura 2). Nenhum apresentou lesão grave. Na fase tardia, 26 (65\%) olhos apresentavam lesão leve (Figura 3) e 14 (35\%) olhos, lesão moderada (Figura 4). Nenhum apresentou lesão grave, isto é, buraco macular.

Os 24 pacientes foram submetidos ao teste com a tela de Amsler para avaliação da função macular, após 6 meses da lesão. Dos 43 olhos, apenas $3(6,9 \%)$ apresentaram escotoma residual.

O teste de sensibilidade ao contraste foi realizado nos 43 olhos e somente o paciente $n^{\circ} 4$ ficou com alteração da acuidade visual de contraste de ambos os olhos (4,6\%).

\section{DISCUSSÃO}

Esta é a maior série de casos (43) de retinopatia solar após ritual religioso. Provavelmente, o número de pessoas que tive-

\begin{tabular}{|c|c|c|c|c|c|c|}
\hline \multicolumn{2}{|c|}{ Final } & \multirow[b]{2}{*}{$20 / 40$} & \multirow[b]{2}{*}{$20 / 30$} & \multirow[b]{2}{*}{$20 / 25$} & \multirow[b]{2}{*}{$20 / 20$} & \multirow[b]{2}{*}{ Total } \\
\hline Inicial & $20 / 70$ & & & & & \\
\hline $20 / 70$ & - & - & - & - & 1 & 1 \\
\hline $20 / 40$ & - & 1 & 1 & 1 & 8 & 11 \\
\hline $20 / 30$ & - & - & 1 & 1 & 7 & 9 \\
\hline $20 / 25$ & - & - & - & 5 & 8 & 13 \\
\hline $20 / 20$ & - & - & - & - & 9 & 9 \\
\hline Total & 0 & 1 & 2 & 7 & 33 & 43 \\
\hline
\end{tabular}

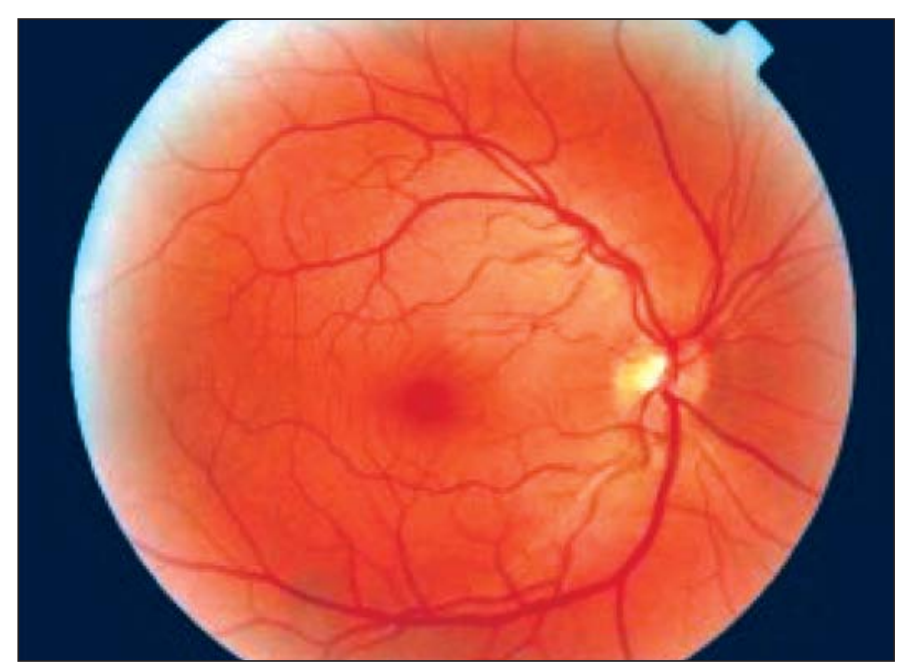

Figura 1 - Maculopatia solar aguda leve

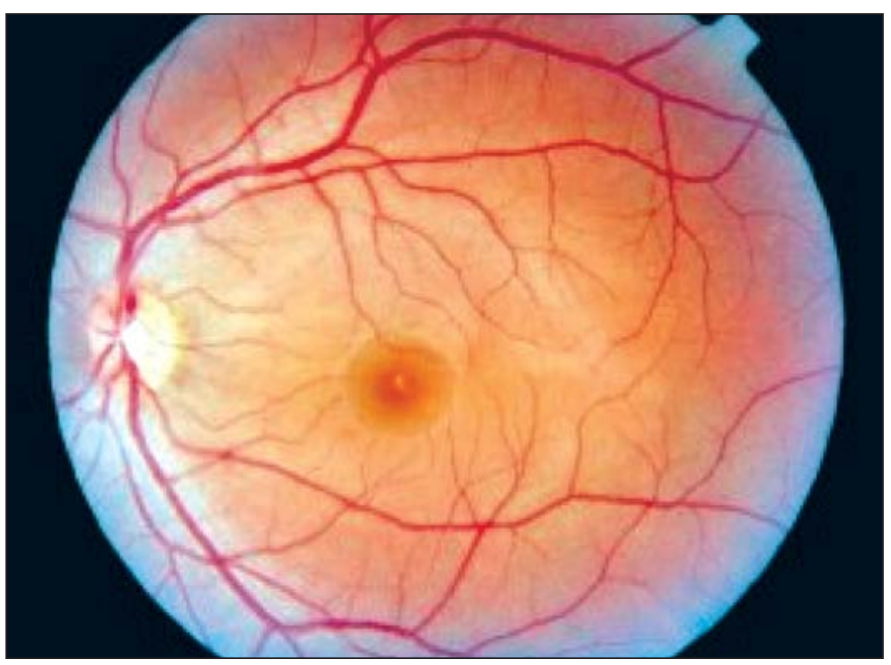

Figura 2 - Maculopatia solar aguda moderada

ram os olhos lesados foi muito maior. Centenas de pessoas participavam de cada sessão deste ritual religioso.

Nos relatos de retinopatia solar o que se verifica é que a sintomatologia se inicia algumas horas após a observação direta do sol. A maioria dos casos apresenta um comprometimento bilateral, embora de forma assimétrica. A visão é reduzida para 20/40 a 20/80, mas pode chegar a 20/200 ou pior em lesões mais severas e surgem sintomas de ofuscamento, escotomas centrais e paracentrais, cefaléia e metamorfopsia. No exame do fundo de olho, imediatamente após a exposição, a mácula pode estar normal. Após 24 horas, há perda do reflexo foveal e em uma semana aparece uma lesão amarelada foveal. Após 14 dias a lesão desaparece nos casos brandos. Porém, em casos moderados a fóvea fica eritematosa com aparência de um cisto avermelhado ou surge uma pequena ( 25 a $50 \mu \mathrm{m}$ ) depressão lamelar (buraco macular) circundado por um anel pigmentado, que é altamente sugestivo de prévio olhar para o sol $^{(12)}$. Em cinco semanas, há resolução do eritema e, em casos 


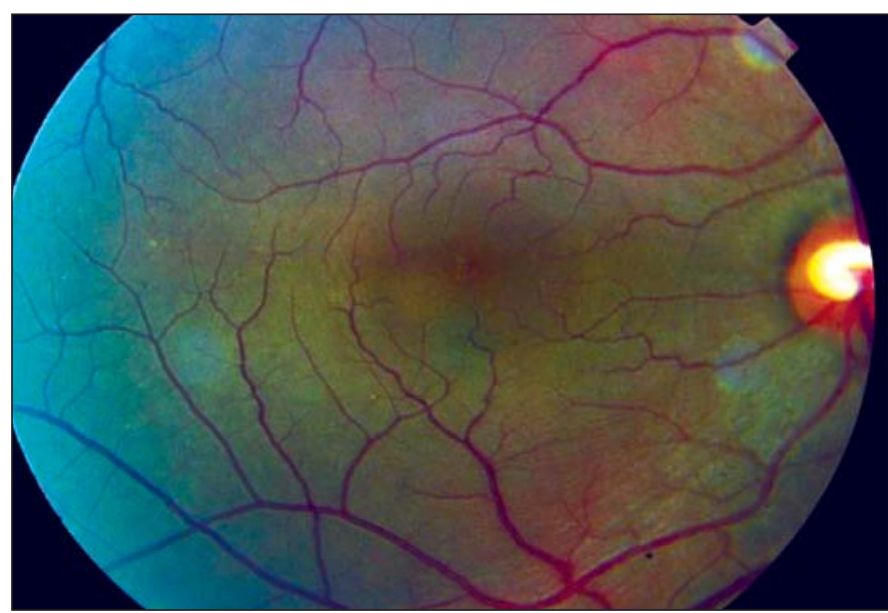

Figura 3 - Maculopatia solar tardia leve

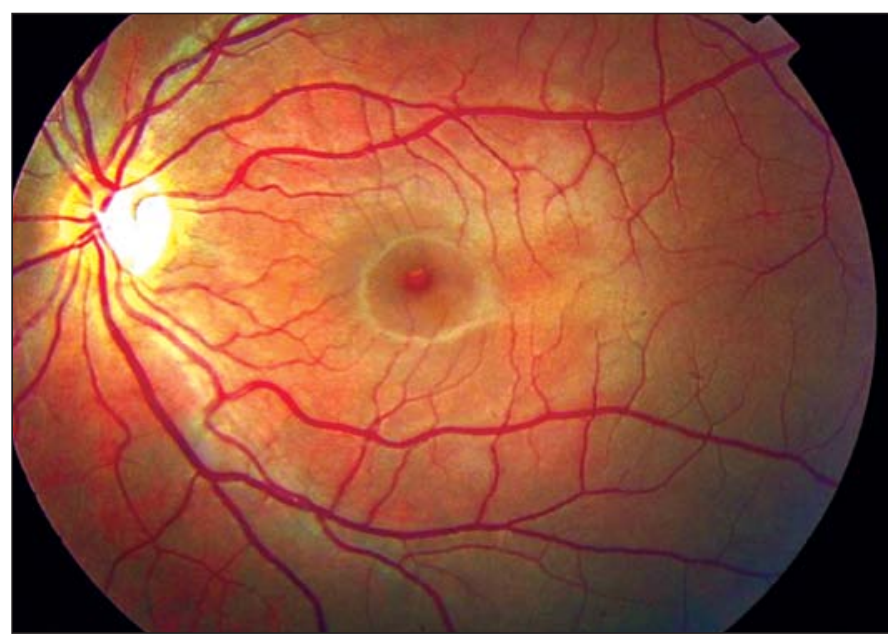

Figura 4 - Maculopatia solar tardia moderada

severos restam algumas irregularidades do epitélio macular e uma pequena área de hipopigmentação. Nos casos de exposições repetidas ao sol, o epitélio pigmentar da retina fica muito alterado e com aspecto de favo de mel. Invariavelmente o vítreo e a retina restante são poupados ${ }^{(13)}$.

O prognóstico visual desta patologia é muito bom. A maioria dos pacientes após algumas semanas, já começam a se recuperar e a visão retorna completamente ou próximo ao normal (20/20 a 20/40), num período de 3 a 9 meses ${ }^{(12)}$.

A recuperação da acuidade visual a 20/20 não quer dizer que houve recuperação total da visão. Alguns pacientes com lesão mais severa permanecem com um escotoma central ou paracentral que vai dificultar mais tarde a leitura e o trabalho de perto. Com o tempo, estes escotomas vão diminuindo e o paciente aprende a fixar excentricamente ${ }^{(14)}$.

Neste estudo, a doença predominou no sexo feminino $(87,5 \%)$ e a idade dos pacientes variou de 18 a 46 anos. Esta ocorrência maior nos jovens pode ser porque esses apresentam um cristalino transparente e por serem mais curiosos $^{(13)}$.

O típico paciente acometido pela retinopatia solar é um adulto jovem, emétrope ou baixo hipermétrope, que foca os raios diretamente sobre a mácula ${ }^{(15)}$.

Dos 43 olhos examinados, 20 (46,6\%) eram hipermétropes, 3 (7\%) míopes, 9 (20,9\%) emétropes e 11 (25,\%) tinham astigmatismo. O equivalente esférico dos graus variou de $-0,25$ a $+0,75$ dioptrias.

O acometimento foi bilateral na maioria dos casos (79,2\%) e na presença de estrabismo e ambliopia a lesão foi limitada ao olho dominante.

A acuidade visual inicial dos pacientes variou de 20/20 a 20/70. Após 6 meses, a acuidade visual foi de 20/25, ou melhor, em 43 (93\%) olhos. Destes, 33 (76,7\%) olhos alcançaram 20/20. Isto mostra que os pacientes tiveram um prognóstico visual bastante favorável, com o retorno da acuidade visual aos níveis pré-exposição.

De acordo com a intensidade das lesões observadas na retinografia na fase inicial, $67,5 \%$ eram leves e $32,5 \%$ moderadas. Após 6 meses, 65\% apresentavam lesões leves e 35\% moderadas. Nenhum paciente apresentou lesão grave na fase inicial e tardia. Apesar de classificada, não foi possível fazer uma correlação entre o aspecto da lesão e a acuidade visual.

Neste estudo, apenas 3 (6,9\%) olhos ficaram com escotoma persistente e $2(4,6 \%)$ com alteração da sensibilidade de contraste.

A retinopatia solar é multifatorial, isto é, depende da exposição, da susceptibilidade, das condições geofísicas e dos fatores fotobiológicos. Até que estudos sejam realizados para elucidar em definitivo como o mecanismo térmico e fotoquímico causam esta doença, o melhor é prevenir. Jamais se deve olhar diretamente para o sol. O modo mais seguro de se observar um eclipse solar é a sua observação indireta, através de uma abertura estenopeica que projeta a imagem do sol em uma tela. Os indivíduos que irão frequentar ambientes muito iluminados e que refletem grande quantidade de luz, como esquiadores, pescadores, velejadores, banhistas e outros, devem ser estimulados a utilizar proteção como chapéus, viseiras e óculos de sol com proteção para raios ultravioleta e antireflexo ${ }^{(16)}$.

As pessoas ainda desconhecem os perigos de se olhar para o sol e isto pode ser confirmado por recentes publicações ${ }^{(17-18)}$.

Nix e Apple (1987), ressaltaram o valor dos relatos de casos de retinopatia solar, para que pessoas sejam desencorajadas a olhar diretamente para o sol. O presente estudo procurou contribuir com este esforço. O relato desses casos foi uma maneira de alertar a população dos perigos da observação direta do sol e avisar da importância das medidas preventivas.

\section{ABSTRACT}

Purpose: To present a series of patients with solar retinopathy after direct solar observation during a religious ritual in Londrina City, Paraná, Brazil. Methods: 24 patients with visual alterations were evaluated after their participation in a religious ritual. Data of the initial and final visit after 6 months of follow-up are presented and include best corrected visual acuity, refraction, color fundus picture, macular biomicrosco- 
py, Amsler's screen and contrast sensibility. Results: A total of 24 cases was reported. Of these, 21 (87.5\%) were females and $3(12.5 \%)$ males. Age varied from 18 to 46 years. A total of 43 eyes were damaged. The lesion was bilateral in 19 (79.2\%) patients and in 5 (20.8\%) it was unilateral. The initial visual acuity ranged from $20 / 20$ to $20 / 70$, and the final visual acuity was 20/25 or better in 40 (93\%) eyes. Of these, $33(76.7 \%)$ eyes reached $20 / 20$ or the last follow-up visit. Only 2 (4.6\%) eyes presented alteration in the sensibility to contrast and $3(6.9 \%)$ remained with persistent scotoma. Conclusion: This is the largest series of solar retinopathy after religious ritual. The cases herein presented showed a quite favorable evolution. However recovery of visual acuity is not complete, once scotoma and residual metamorphopsia persisted in a few patients.

Keywords: Eye/pathology; Retina/pathology; Visual acuity/radiation effects Sunlight/adverse effects; Cerimonial behavior

\section{REFERÊNCIAS}

1. Istock TH. Solar retinopathy: a review of the literature and case report. J Am Optom Assoc 1985;56:374-82.

2. Stokkermans TJ, Dunbar MT. Solar retinopathy in a hospital-based primary care clinic. J Am Optom Assoc 1998;69:625-36.
3. Hope-Ross M, Travers S, Mooney D. Solar retinopathy following religious rituals. Br J Ophthalmol 1988;72:931-4.

4. Suzukim CR, Taba KE, Ramalho AML, Almeida GV. Maculopatia solar: relato de seis casos. Arq Bras Oftalmol 1997;60:16-20.

5. Wergeland FL Jr, Brenner EH. Solar retinopathy and foveomacular retinitis. Ann Ophthalmol 1975;7:495-8,501-3.

6. Flynn JAF. Retinal burns after sun’s eclipse. Br Med J 1960;563.

7. Gladstone GJ, Tasman W. Solar retinitis after minimal exposure. Arch Ophthalmol 1978;96:1368-9.

8. Ewald RA. Sun gazing associated with the use of LSD. Ann Ophthalmol 1971;3:15-7.

9. Galainena ML. Solar retinopathy. Ann Ophthalmol 1976,8:304-6.

10. Cangelosi GC, Newsome DA. Solar retinopathy in persons on religious pilgrimage. Am J Ophthalmol 1988;105:95-7.

11. Nix RR, Apple DJ. Solar retinopathy from sungazing in Medjugorje. J La State Med Soc 1987;139:36-40.

12. Gass JD. Stereoscopic atlas of macular diseases: diagnosis and treatment. $3^{\text {rd }}$ ed. St Louis: Mosby;1987. p.570-2.

13. Dhir SP, Gupta A, Jain IS. Eclipse retinopathy. Br J Ophthalmol 1981;65:42-5.

14. Pang HG. Eclipse retinopathy. Am J Ophthalmol 1963;55:383-4.

15. Yannuzzi LA, Fisher YL, Slakter JS, Krueger A. Solar retinopathy. A photobiologic and geophysical analysis. Retina 1989;9:28-43.

16. Zisman M, Nehemy MB. Retinopatia solar: relato de 5 casos. Rev Bras Oftalmol 1995;54:43-8.

17. Mwanza JC, Kayembe DL, Kaimbo DK, Kabasele PM, Ngoy DK. Solar retinopathy acquired after gazing at the sun during prayers. Bull Soc Belge Ophtalmol 2000;275:41-5.

18. Wong SC, Eke T, Ziakas NG. Eclipse burns: a prospective study of solar retinopathy following the 1999 solar eclipse. Lancet 2001;357:199-200.

\section{$5^{\circ}$ Curso Internacional de Glaucoma}

\section{4 e 15 de Maio de 2004 Maksoud Plaza Hołel - SP}

INFORMAÇÕES: JDE Comunicação e Eventos

Tel.: (1 1 ) 287-9378 / 289-4301

Fax: (1 1) 288-8157

E-mail: jdecomev@uol.com.br 